\title{
A Socio-Technical, Probabilistic Risk Assessment Model for Surgical Site Infections in Ambulatory Surgery Centers
}

\author{
Ebru K. Bish, PhD; ${ }^{1}$ Hadi El-Amine, MS; ${ }^{1}$ Laura A. Steighner, PhD $;^{2}$ Anthony D. Slonim, MD ${ }^{3}$
}

\begin{abstract}
BACKGROUND. To understand how structural and process elements may affect the risk for surgical site infections (SSIs) in the ambulatory surgery center (ASC) environment, the researchers employed a tool known as socio-technical probabilistic risk assessment (ST-PRA). STPRA is particularly helpful for estimating risks in outcomes that are very rare, such as the risk of SSI in ASCs.
\end{abstract}

овJECTIVE. Study objectives were to (1) identify the risk factors associated with SSIs resulting from procedures performed at ASCS and (2) design an intervention to mitigate the likelihood of SSIs for the most common risk factors that were identified by the ST-PRA for a particular surgical procedure.

METHODS. ST-PRA was used to study the SSI risk in the ASC setting. Both quantitative and qualitative data sources were utilized, and sensitivity analysis was performed to ensure the robustness of the results.

RESULTS. The event entitled "fail to protect the patient effectively" accounted for $51.9 \%$ of SSIs in the ambulatory care setting. Critical components of this event included several failure risk points related to skin preparation, antibiotic administration, staff training, proper response to glove punctures during surgery, and adherence to surgical preparation rules related to the wearing of jewelry, watches, and artificial nails. Assuming a 75\% reduction in noncompliance on any combination of 2 of these 5 components, the risk for an SSI decreased from 0.0044 to between 0.0027 and 0.0035 .

CONCLUSION. An intervention that targeted the 5 major components of the major risk point was proposed, and its implications were discussed.

Infect Control Hosp Epidemiol 2014;35(S3):S133-S141

Outpatient procedures represent more than three-quarters of all operations performed in the United States and are performed increasingly for patients whose conditions are more complex and who have more comorbid conditions..$^{1-3}$ In recent years, there has been a significant increase in the number of ambulatory surgery centers (ASCs) nationally and an expansion of the volume, types, and complexity of procedures performed at these centers. ${ }^{4}$ Given the increasing number of ASCs, expansion of the procedures performed at ASCs, and the variability in their ownership and organizational structure, it is important to understand healthcare-associated infection (HAI) rates, their risk factors, and how they differ for ASCs compared with hospitals to improve the care of patients in this context. Surgical site infection (SSI) is the most common HAI among surgical patients ${ }^{5}$ and is the focus of this research effort.

The socio-technical probabilistic risk assessment (ST-PRA) is an engineering tool commonly used for the modeling and analysis of complex engineering systems, with the goals of quantifying the risk of high-impact, low-probability events ("adverse events") and identifying improvement actions ("interventions") for reducing the risk..$^{6-8}$ ST-PRA is an ideal tool for studying the risk of SSIs arising in the ASC setting, because the processes at ASCs and possible failure points depend on the interactions between people, systems, and technology. Furthermore, SSIs fit perfectly within the definition of highimpact, low-probability events. Consequently, we performed an ST-PRA to map the cause-and-effect relationships between failure points and SSI risk in the ASC setting and to identify improvement actions.

\section{METHODS}

\section{Definition of SSI}

The Centers for Disease Control and Prevention definition of an SSI is an infection that occurs after a requisite procedure that involves a surgical incision and is performed in an operating room setting.

Affiliations: 1. Grado Department of Industrial and Systems Engineering, Virginia Tech, Blacksburg, Virginia; 2. American Institutes for Research, Washington, DC; 3. Renown Health, Reno, Nevada.

Received March 14, 2014; accepted April 20, 2014; electronically published September 15, 2014

(C) 2014 by The Society for Healthcare Epidemiology of America. All rights reserved. 0899-823X/2014/35S3-0019\$15.00. DOI: 10.1086/677824 


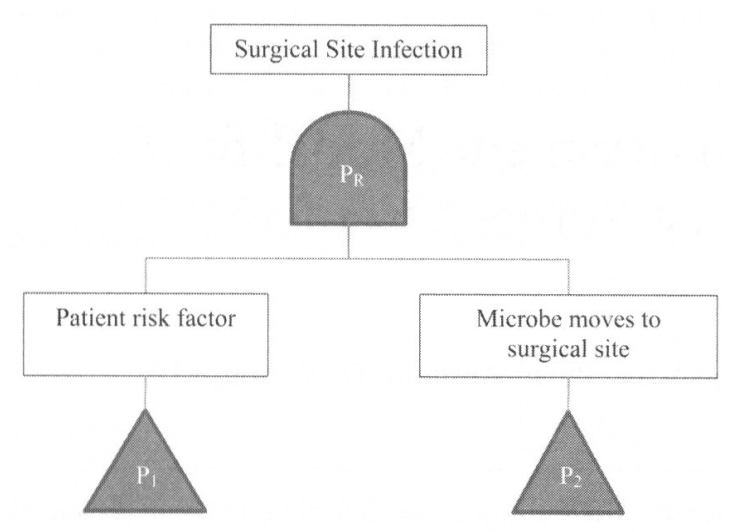

FIGURE 1. Top event "surgical site infection" with 2 lower-level events, "patient risk factor" and "microbe moves to surgical site."

\section{Surgical Procedure}

The study was restricted to arthroscopic knee surgery, which was selected because it is a high-volume procedure, is performed across all age groups, and is amenable to an ambulatory intervention..$^{10}$

\section{Setting and Partners}

Our clinical partners included 4 ASCs, each representing a different care context: an academic hospital-associated ASC; a community hospital-associated ASC; a free-standing forprofit ASC; and a free-standing, hospital-associated, pediatric ASC. A technical expert panel (TEP), consisting of $12 \mathrm{mem}$ bers, acted in an advisory capacity for the researchers to clinically validate the assessment and interventions.

\section{ST-PRA Methodology}

ST-PRA is a powerful risk assessment and management tool for several reasons. (1) It proactively identifies a system's risk points ("failure points" or "basic events") before failures occur. (2) It systematically models complex interactions among people, processes, and technology through the use of deductive logic, Boolean algebraic, and probability theory, allowing the construction of a model ("fault tree") of how risk points interact with one another, either individually or collectively, to contribute to the adverse event of interest. As a result, the ST-PRA methodology is especially well-suited for healthcare systems in which multiple failures, often involving a combination of people, processes, and technology, must occur for an adverse event to occur. (3) It uses both quantitative and qualitative data from various sources (eg, risk estimates from the evidence-based literature and experiential estimates from system users) to "map" the risks associated with the adverse event in a robust manner. (4) It quantifies the risk of the adverse event under investigation and identifies the failure points that are the highest contributors to the adverse event. As such, it provides a road map for practitioners in the form of a prioritized list of interventions that can reduce risk the most.

ST-PRA methodology involves 6 steps; detailed descriptions regarding the approach to modeling have been published elsewhere. ${ }^{11-13}$ (1) Identify the adverse event of interest ("top event"). (2) Identify all failure points or basic events that may independently or in conjunction with each other contribute to the top event. (3) Identify the dependencies and interactions among the risk points, creating a fault tree that depicts the relationships and interconnections between the

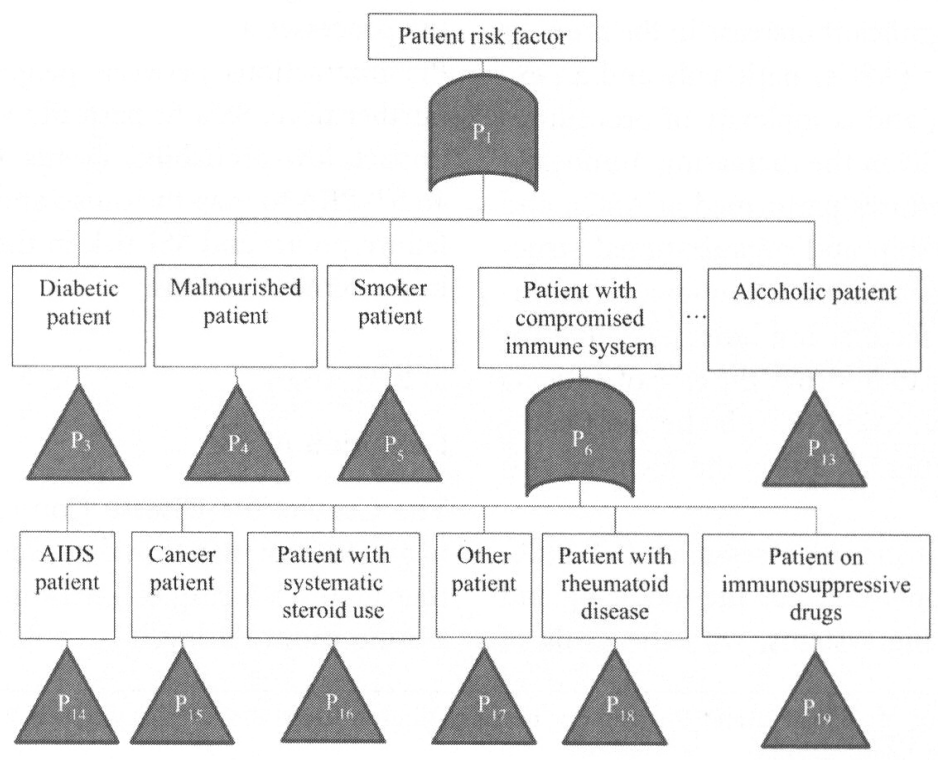

FIGURE 2. Subtree for event "patient risk factor" with children nodes presented for the lower-level event "patient with compromised immune system." 


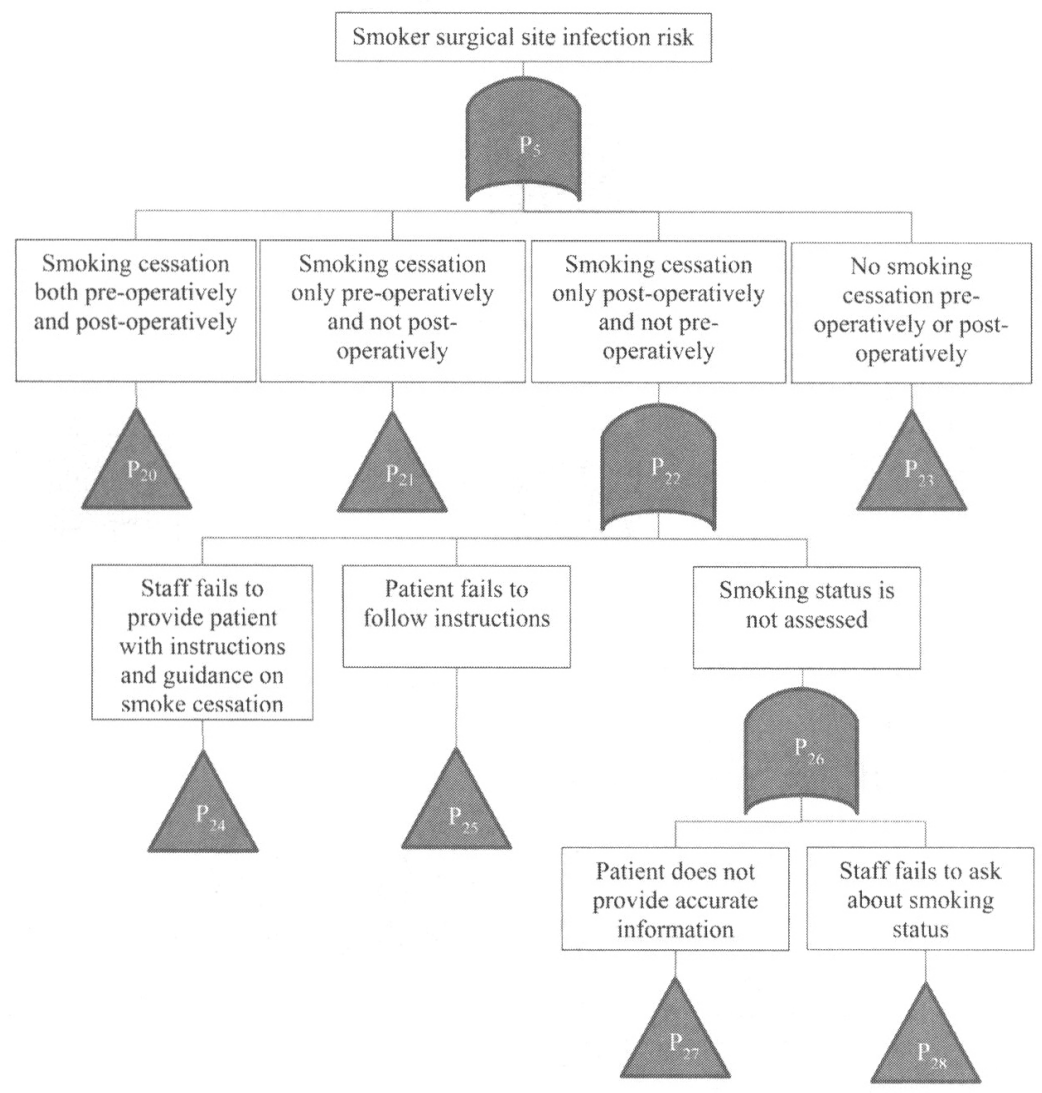

FIGURE 3. Subtree for event "smoker surgical site infection risk" with children nodes presented for the lower-level event "smoking cessation only postoperatively and not preoperatively."

basic events using various logical operators. (4) Validate the structure and logic of the fault tree model. (5) Identify the likelihood of the basic events in the fault tree model. (6) Conduct a sensitivity analysis of the fault tree model by varying the range of probability values.

\section{Data Sources Used}

The following data sources were used in this research to ensure that the fault tree captured all possible risk points and patient factors: (1) the peer-reviewed and gray literature, (2) national databases, (3) ASC site visits, and (4) TEP input. Each source informed the data collection effort for the other sources in an iterative fashion. That is, information gleaned during the literature review informed ways to analyze the databases; information collected during the site visits or during the TEP meetings informed additional data analyses and literature searches.

We used Relex Reliability Studio 2007 software, version 2, to identify the failure points that are the top contributors to SSI risk and to determine the corresponding SSI risk. We conducted a sensitivity analysis by varying a set of basic event probabilities over specific ranges to test the model's robustness. Please see Slonim et $\mathrm{al}^{11}$ and Bish et $\mathrm{al}^{13}$ for details on the analysis.

\section{RESULTS}

\section{Construction of the Fault Tree}

The top event of interest for this research is an SSI occurring in an ASC setting. Relevant literature suggests the following conceptual model: "Microbial contamination of the surgical site is a necessary precursor of SSI. The risk of SSI can be conceptualized according to the following relationship: risk of SSI $=$ (dose of bacterial contamination $\times$ virulence $) /$ resistance of the host patient."14(p102)

Two conditions must be met for an SSI to occur: (1) the surgical site must be contaminated with the microbe, and (2) the host patient's resistance should be low enough to enable infection to occur. In other words, the contamination of the surgical site with the microbe is necessary but not sufficient for an SSI to develop; patients with some risk factors are more likely to develop an SSI after an encounter with the microbe. $^{15}$

We model this phenomenon in the fault tree by assuming that the patient either acquires the microbe inside of the ASC because of a system failure (eg, failure to sterilize equipment appropriately and failure to clean high-touch areas appropriately) or the patient enters the ASC with the microbe and remains with the microbe due to a system failure (eg, failure 


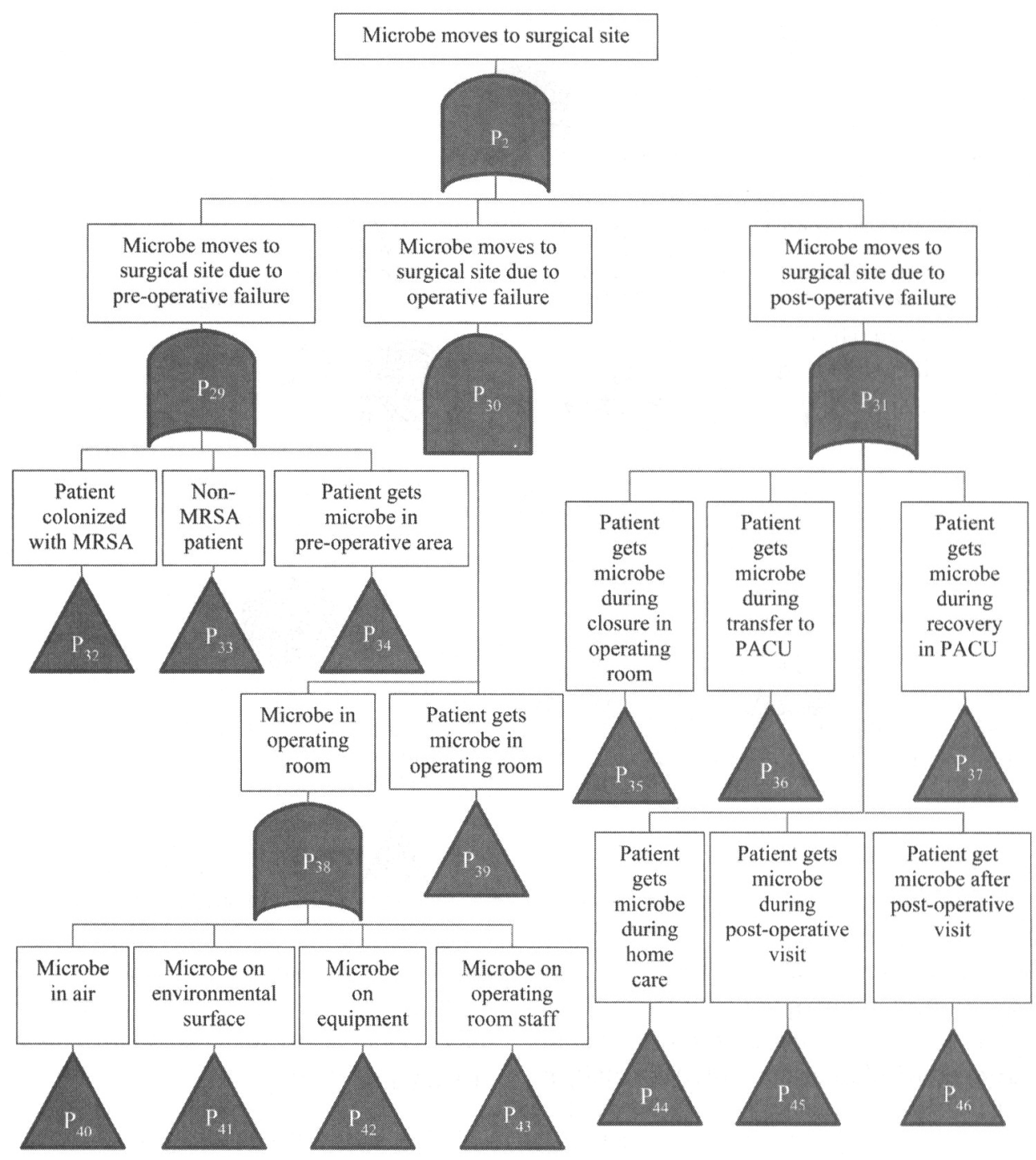

FIGURE 4. Subtree for event "microbe moves to the surgical site" with children nodes presented for all lower-level events. MRSA, methicillinresistant Staphylococcus aureus. PACU, postanesthesia care unit.

to screen and identify a methicillin-resistant Staphylococcus aureus [MRSA]-infected patient). Therefore, we link (1) microbial contamination to a set of failures in the system and (2) host patient's resistance to patient characteristics (risk factors) and failures to mitigate them. This allows patient risk factors and system failure points to be incorporated into risk estimates in the fault tree. The fault tree incorporates system failures that may occur during the preoperative, operative, and postoperative phases.

The final fault tree contained 283 events and 203 gates and can be found in Slonim et al. ${ }^{12}$ In this article, we provide select examples from the fault tree and show only parts of the fault tree to demonstrate our modeling approach. In the figures, an "AND" gate is represented by the single arched window symbol, and an "OR" gate is represented by the double-arched window symbol. The probabilities of events from lower levels of the tree are displayed inside the triangles.
Figure 1 depicts the top part of the fault tree in which the occurrence of an SSI event depends on the occurrence of 2 lower-level events, "patient risk factor" and "microbe moves to surgical site." Given the patient's encounter with the microbe, the likelihood of an SSI will depend on the patient's risk factor (if any). Therefore, these 2 events are connected using an AND gate. The term $\mathrm{P}_{\mathrm{r}}$ corresponds to the probability of an SSI and is calculated using the probabilities of the basic events (provided by the ST-PRA modeler) and the logical relationships in the tree.

Next, we present our modeling approach for the node, "patient risk factor," depicted in Figure 1. (A node is an "event" or risk point; the terms are used interchangeably. During model building, "node" is the common term used.) The event "patient risk factor" can be further expanded, via an OR gate, into its "children nodes" (events), each of which represents a specific risk factor, as depicted in Figure 2. Patient 


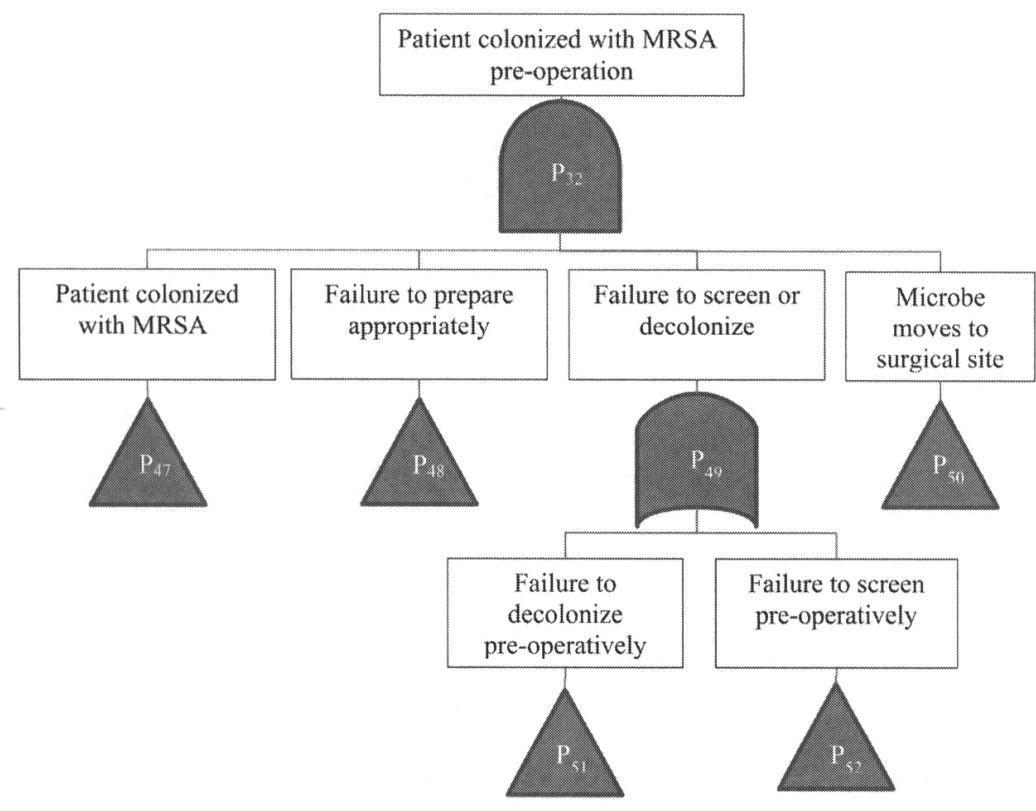

FIGURE 5. Subtree for event "patient colonized with MRSA preoperation" with children nodes presented for the lower-level event "failure to screen or decolonize." MRSA, methicillin-resistant Staphylococcus aureus.

risk factors explicitly modeled in the fault tree include diabetic, malnourished, obese, smoker, alcoholic, and elderly; patient with vascular insufficiency, skin lesion, compromised immune system, or high temperature; and otherwise healthy patient. We also considered the intersection of these events, that is, cases in which a patient might be both obese and diabetic, for example. Some of these risk factors are further detailed into their children nodes, such as Figure 2, which depicts the children nodes for "patient with compromised immune system."

For each risk factor, the risk could potentially be mitigated during the preoperative phase. If a risk factor is mitigated (eg, a smoker patient is instructed to stop smoking prior to surgery and the patient follows instructions and stops smoking), then the SSI risk of that patient will be reduced. Figure 3 illustrates this modeling approach for the smoker patient. A similar approach is used in the fault tree to model preoperative mitigation opportunities for all children nodes "patient risk factor." That is, each of these children nodes corresponds to an "intermediate node," which is built up from lower level events in the tree and connected all the way to basic events. Continuing with our example, a smoker patient may or may not adhere to preoperative and postoperative instructions for smoking cessation, leading to 4 mutually exclusive events, all connected with an OR gate: (1) smoking cessation both preoperatively and postoperatively; (2) smoking cessation only preoperatively and not postoperatively (the patient resumes smoking postoperatively); (3) smoking cessation only postoperatively and not preoperatively (the patient does not stop smoking preoperatively), and no smoking cessation preoperatively or postoperatively.
In Figure 3, we also provide the subtree for the event "smoke cessation only postoperation," obtained by expanding this node, via an OR gate, into its children nodes: "staff fails to provide patient with instructions and guidance on smoke cessation," "patient fails to follow instructions," and "smoke status is not assessed." The event "smoke status is not assessed" is further connected via an OR gate to its children nodes, "patient does not provide accurate information" and "staff fails to ask about smoking status."

In Figure 4, we discuss our modeling approach for the intermediate event, "microbe moves to surgical site," first noted in Figure 1. Each of the children nodes of this event, in turn, represents an intermediate event. As Figure 4 demonstrates, the fault tree incorporates system failures occurring during the preoperative, operative, and postoperative phases. Next, we provide an example of how failures in each phase are modeled in the fault tree.

As a preoperative example, consider the intermediate event "microbe moves to surgical site due to preoperative failure" in Figure 4. System failures that need to occur for this event may differ for patients entering the ASC with MRSA (represented by the node "patient colonized with MRSA"), for patients that capture the microbe in the preoperative wait area (represented by the node, "patient gets microbe in preoperative area"), and for other patients (represented by the node, "non-MRSA patient"). Each of these nodes is modeled further in the fault tree. For example, Figure 5 depicts the expansion of the node "patient colonized with MRSA" to its children nodes, and Figure 6 depicts the further expansion of the node "failure to decolonize the patient preoperatively" to its children nodes. 


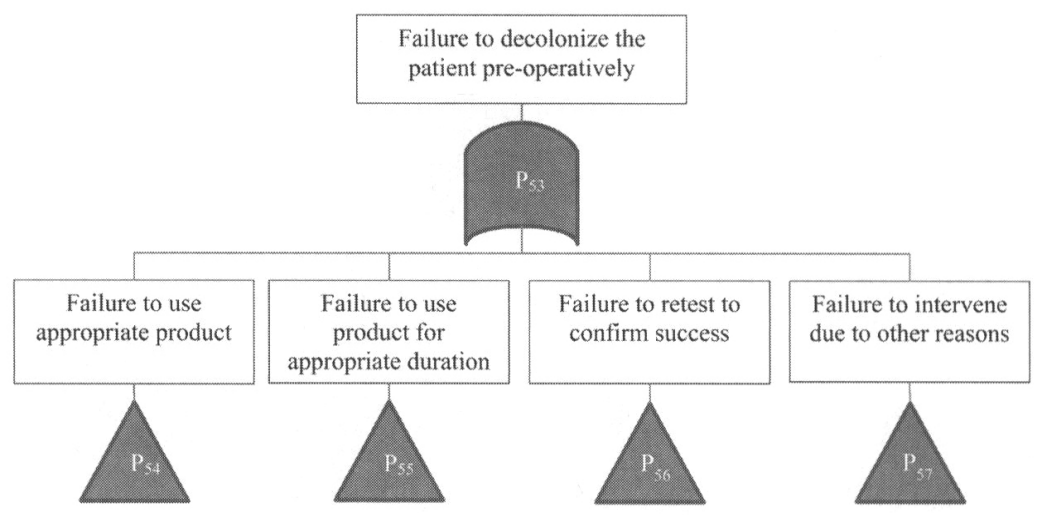

Figure 6. Subtree for event "failure to decolonize the patient preoperatively" with all lower-level events.

Similarly, for the operative phase for the intermediate event "microbe moves to surgical site due to operative failure" to occur during surgery, the microbe needs to be present in the operating room (represented by the node, "microbe in operating room"), and the microbe needs to get to the patient (represented by the node, "patient gets microbe in operating room"), as depicted in Figure 4. Figure 4 also expands the node "microbe in the operating room" to its children nodes ("microbe in air," "microbe on environmental surface," "microbe on equipment," and "microbe on operating room staff"), each of which is further expanded into its own children nodes. Figure 7 expands the node "patient gets microbe during home care" depicted in Figure 4 to its children nodes ("failure to follow postoperative directions" and "failure to notice or manage"), which are further expanded into their own children nodes.

\section{Events Targeted for Intervention}

The original risk of developing an SSI is estimated by the fault tree model as 0.0044 (ie, 44 out of 10,000 cases will develop an SSI following surgery). "Event 642: Failure to effectively protect patient" and its component events (children nodes) accounted for $51.9 \%$ of SSIs in the ASC setting. The most important components that comprise this failure, as indicated by their contribution to the risk of developing an SSI, along with their labels in the fault tree, include (1) failure to prepare the skin appropriately preoperatively (event 30); (2) antibiotic related failure (gate 239); (3) staff not welltrained in infection control practices (event 142); (4) glove puncture (event 659); and (5) failure to remove watch, jewelry, or artificial nails (event 138).

Because improvement efforts can never be $100 \%$ successful at mitigating risk, we examined the variable impact of an intervention using values of $25 \%, 50 \%$, and $75 \%$ reduction in noncompliance rates. For example, if an ASC chooses to focus on improving skin preparation practices, the intervention would reduce the likelihood of that risk factor (ie, event 30: "failure to prepare the skin appropriately") from its original value of 0.125 to 0.0625 if the noncompliance rate was reduced by $50 \%$. The combined impact on the risk of developing an SSI for each of these combinations at different impact levels is presented in Table 1. For example, if the ASC selected interventions that targeted both the failure to prepare the skin appropriately (event 30) and training for staff in infection control practices (event 142) and expected only a $25 \%$ reduction in noncompliance rate for each, the probability of an SSI would actually be further reduced to 0.0039 .

\section{I S CUSSION}

The 3 key factors taken into consideration during the intervention design phase were (1) ease of implementation, (2) the likelihood of achieving substantive improvement, and (3) the level of effort necessary for its effective implementation within an existing system (ie, ASC environment). As the results of the fault tree analysis indicate, the event targeted for an intervention was "event 642: failure to effectively protect patient" (with its children nodes detailed earlier).

Interestingly, several of these components are included as infection control requirements by the Joint Commission or the Centers for Medicare and Medicaid Services accreditation and compliance processes in the hospital surgical environment (ie, there are specific infection control standards regarding the wearing of jewelry and artificial nails, use of antibacterials, and other associated infection control practices). Although not required in ASCs, some facilities have adopted these policies. For example, hospital-affiliated ASCs tend to incorporate their hospital's policies as their own; however, there can be great variability in how the policies and procedures are monitored and enforced, further highlighting this set of events as the target for a potentially valuable intervention.

The proposed intervention included developing and instituting guidelines for infection control practices at ASCs, modeled after the guidelines provided to hospitals and including policies and procedures for the prevention of preoperative infection transmission (eg, hygiene, infectious waste, personal protective equipment, infectious patients, prevention of 


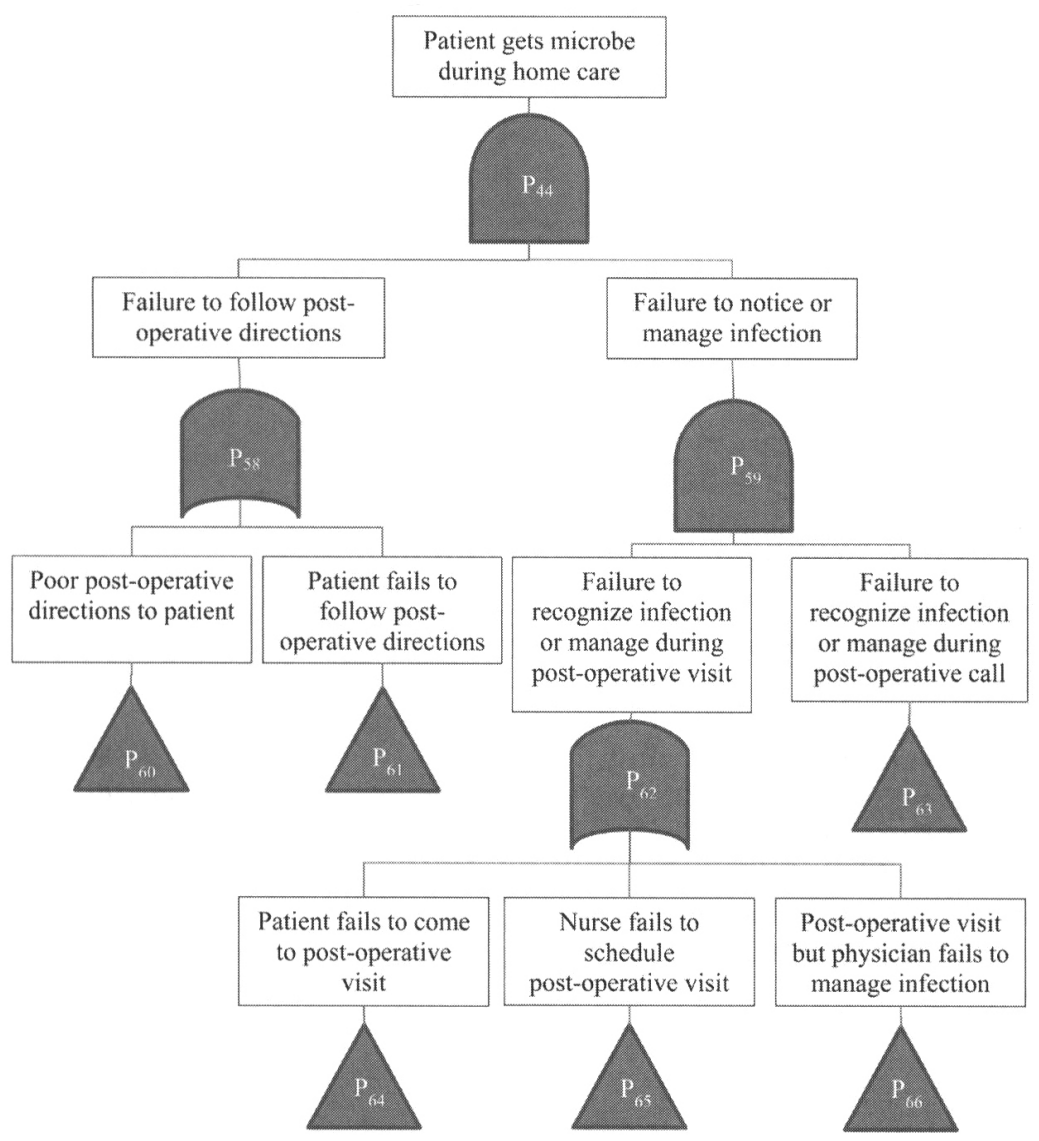

FIGURE 7. Subtree for event "patient gets microbe during home care" with children nodes presented for all lower-level events.

patient-to-patient transmission, assessment of risk factors for SSIs, and risk procedures), prevention of intraoperative infection transmission (eg, surgical disinfection and antisepsis, skin preparation, and disinfection in the surgical environment), and prevention of postoperative infection transmission (eg, dressing the postoperative wound).

The intervention should also include opportunities for (1) staff training on the proper technique to ensure consistency across providers and personnel and (2) observation of staff technique in the ASC environment. We also recommended that a bundle addressing the following issues be identified: (1) identification of high-risk patients (eg, patients with diabetes, MRSA, or obesity); (2) procedures to ensure removal of jewelry, watches, and artificial nails; (3) guidance on routine double gloving and preoperation response to glove punctures; (4) skin preparation practices including antisepsis and draping; and (5) proper administration and timing of antibacterial agents.

In this study, we used the ST-PRA to identify the contributors to the occurrence of SSIs in the ASC environment. The value of ST-PRA lies in the capacity to consider both individual contributors of risk and unique combinations of risks that contribute to the adverse outcome. By including both quantitative and qualitative data in the models, a real-world experience can be created and tested using the sensitivity analysis methodology, allowing the ST-PRA modeler to maintain the scientific integrity of the tool. Finally, the ST-PRA model also serves as a living document that can continue to be modified over time as new risk information is acquired either through direct observation or improved methods for studying the ASC environment.

Despite these important strengths, notable limitations of this study should be acknowledged. The lack of integrated data systems, linking patients between the ASC, emergency department, and inpatient settings, significantly limited the ability to inform the model with real risk estimates. Until these linkages can be established, investigators studying care in the ASC setting will continually face the challenge of "loss to follow-up." Patients tend to seek care for surgical complications in emergency departments, inpatient settings, or their primary care physicians' offices, and the attribution of the complication may not return to the ASC where it originated. Although a typical limitation of such modeling exercises is that the resulting models do not create a real world picture of what it is intended to portray, this was not a limitation of the current study. The use of quantitative estimates from the literature and the modeling of the in vivo process flows contributed to a real life understanding of the system 
TABLE 1. Probability of Surgical Site Infection (SSI) by Multiple Concurrent Interventions at Various Reductions of Noncompliance Rates (25\%, 50\%, and 75\%)

\begin{tabular}{|c|c|c|c|c|c|}
\hline Event/gate & Event 30 & Gate 239 & Event 142 & Event 659 & Event 138 \\
\hline \multicolumn{6}{|l|}{$25 \%$ reduction in noncompliance } \\
\hline $\begin{array}{l}\text { Event } 30 \text { : fail to prepare skin } \\
\text { appropriately }\end{array}$ & .0042 & .0038 & .0039 & .0040 & .0041 \\
\hline \multicolumn{6}{|l|}{ Gate 239: antibiotic-related } \\
\hline failure & $\ldots$ & .0040 & .0037 & .0038 & .0039 \\
\hline \multicolumn{6}{|l|}{ Event 142: staff not well } \\
\hline trained in infection control & $\ldots$ & $\ldots$ & .0041 & .0039 & .0039 \\
\hline Event 659: glove puncture & $\ldots$ & $\ldots$ & $\ldots$ & .0042 & .0041 \\
\hline \multicolumn{6}{|l|}{ Event 138: fail to remove } \\
\hline \multicolumn{6}{|l|}{$50 \%$ reduction in noncompliance } \\
\hline \multicolumn{6}{|l|}{ Event 30 : fail to prepare skin } \\
\hline \multicolumn{6}{|l|}{ Gate 239: antibiotics-related } \\
\hline \multicolumn{5}{|l|}{ Event $142:$ staff not well } & .0035 \\
\hline Event 659: glove puncture & $\ldots$ & $\ldots$ & $\ldots$ & .0040 & .0037 \\
\hline \multicolumn{5}{|l|}{ Event 138: fail to remove } & .0041 \\
\hline \multicolumn{6}{|l|}{$75 \%$ reduction in noncompliance } \\
\hline \multicolumn{6}{|l|}{ Event 30: fail to prepare skin } \\
\hline \multicolumn{6}{|l|}{ Gate 239: antibiotics-related } \\
\hline \multicolumn{6}{|l|}{ Event 142: staff not well } \\
\hline $\begin{array}{l}\text { Event 659: glove puncture } \\
\text { Event 138: fail to remove }\end{array}$ & $\ldots$ & $\ldots$ & $\ldots$ & .0039 & .0034 \\
\hline watch/jewelry/fake nails & $\ldots$ & $\ldots$ & $\ldots$ & $\ldots$ & .0039 \\
\hline
\end{tabular}

NOTE. An "event" represents a risk point, and a "gate" represents a logical combination of a series of events. The numbers in the table represent the probability of an SSI occurring when multiple interventions are performed with varying rate reduction in noncompliance with the intervention. For example, a $25 \%$ reduction in noncompliance rate for "fail to prepare skin appropriately" and "staff not well-trained in infection control" events simultaneously reduces to the SSI risk from its original probability of .0044 to .0039 .

under study when combined with the sensitivity analyses, which ensured that the risk estimates and conclusions were supported across a range of values.

\section{ACKNOWLEDGMENTS}

Financial support. This project was funded under contract number HHSA290200600019I Task Order 12 from the Agency for Healthcare Research and Quality (AHRQ), US Department of Health and Human Services. The opinions expressed in this document are those of the authors and do not reflect the official position of AHRQ or the US Department of Health and Human Services.

Potential conflicts of interest. The authors confirm that we have no other relevant relationships, conditions, or circumstances that present a potential conflict of interest. All authors submitted the ICMJE Form for Disclosure of Potential Conflicts of Interest, and the conflicts that the editors consider relevant to this article are disclosed here.

Address correspondence to Laura Steighner, $\mathrm{PhD}$, American Institutes for Research, 1000 Thomas Jefferson Street NW, Washington, DC 20007 (lsteighner@air.org).

\section{REFERENCES}

1. Barie P. Infection control practices in ambulatory surgical centers. I Am Med Assoc 2010;303:2295-2297.

2. Centers for Disease Control and Prevention. U.S. outpatient surgeries on the rise. http://www.cdc.gov/media/pressrel/2009 /r090128.htm. Published January 28, 2009. Accessed November 14, 2013.

3. Ambulatory Surgery Center Association. For patients: what is an ASC? http://www.ascassociation.org/asca/resourcecenter /forpatients/. Accessed April 16, 2014. 
4. Hall M, Lawrence L. Ambulatory surgery in the United States: US Department of Health and Human Services, Centers for Disease Control and Prevention, National Center for Health Statistics, 1996, 1997.

5. Hedrick TL, Anastacio MM, Sawyer RG. Prevention of surgical site infections. Expert Rev Anti Infect Ther 2006;4:223-233.

6. Slonim A, Bish E, Xie R. Red blood cell transfusion safety: probabilistic risk assessment and cost/benefits of risk reduction strategies. Ann Oper Res 2011:1-30.

7. Cohen M, Smetzer J, Westphal J, Comden S. Risk models to improve safety of dispensing high-alert medications in community pharmacies. J Am Pharm Assoc 2012;52:584-602.

8. Bish D, Bish E, Xie R, Slonim A. Optimal selection of screening assays for infectious agents in donated blood. IIE Trans Healthc Syst Eng 2011;1:67-90.

9. Centers for Disease Control and Prevention. Surgical Site Infection (SSI). http://www.cdc.gov/HAI/ssi/ssi.html. Accessed February 4, 2014.

10. Agency for Healthcare Research and Quality. California State Ambulatory Surgery Database. 2008. http://www.hcup-us.ahrq .gov/sasdoverview.jsp.

11. Slonim A, Bish E, Steighner L, Zeng X, Crossno R. Proactive risk assessment of surgical site infections in ambulatory surgery centers: final report (prepared by the American Institutes of Research under contract no. 290-06-00019i-12). Agency for Healthcare Research and Quality (AHRQ) publications no. 120045-ER. Rockville, MD: Agency for Healthcare Research and Quality, 2012.

12. Slonim A, Bish E, Steighner L. Using socio-technical probabilistic risk assessment (ST-PRA) to assess risk and improve patient safety and reliability in healthcare systems. In: Battles JB, Cleeman JI, Kahn KL, Weinberg DA, eds. Advances in the Prevention and Control of HAIs. Rockville, MD: Agency for Healthcare Research and Quality, 2014.

13. Bish E, Azabeh-Fard N, Steighner L, Hall K, Slonim A. Proactive risk assessment of surgical site infections in ambulatory surgery centers. J Patient Saf (forthcoming).

14. Mangram A, Horan T, Pearson M, Silver LC, Jarvis WR; Hospital Infection Control Practices Advisory Committee. Guideline for prevention of surgical site infection, 1999. Am J Infect Control 1999;27:97-134.

15. Barie P, Eachempati S. Surgical site infections. Surg Clin North Am 2005;85:1115-1135. 